\title{
LA CATEGORÍA DE MODELACIÓN Y EL CONCEPTO DE INTEGRAL DEFINIDA: UNA MIRADA SOCIOEPISTEMOLÓGICA
}

\author{
THE MODELING CATEGORY AND THE CONCEPT OF \\ DEFINITE INTEGRAL: A SOCIOEPISTEMOLOGICAL VIEW
}

\author{
CLAUDIO GAETE PERALTA*
}

Rec.: 05-11-2019 Acept:: 29-05-2020 Publ.: 30-jun-2020

http://doi.org/10.29035/ucmaule.58.83

\section{RESUMEN}

El discurso Matemático Escolar opaca los usos de la acumulación que ocurren en otros dominios de conocimiento y en el cotidiano de la gente. Enmarcada en la teoría socioepistemológica, la presente investigación tuvo como objetivo desarrollar la categoría de modelación como una resignificación de usos de la acumulación en dos situaciones específicas de variación, una perteneciente al dominio de la Fenología y la otra al dominio de la Economía. Dicho desarrollo permite dar bases para el diseño de situaciones escolares de socialización en educación superior cuya finalidad sea incorporar dichos usos a los procesos de construcción del concepto de integral definida, considerando la funcionalidad del conocimiento matemático, la pluralidad epistemológica y la transversalidad del saber.

Palabras clave: Categoría de Modelación, Economía, Fenología, Socioepistemología, Usos de la acumulación.

\section{ABSTRACT}

School Mathematical discourse obscures the uses of accumulation that occur in other domains of knowledge and in people's daily lives. Framed in socioepistemological theory, this research aimed to develop the modeling category as a resignification of uses of accumulation in two specific situations of variation, one belonging to the domain of Phenology and the other to the domain of Economy. Said development allows

* Doctor y Magíster en Didáctica de la Matemática. Magíster y Licenciado en Matemáticas. Pontificia Universidad Católica de Valparaíso. Jefe Departamento de Matemáticas y Física. Facultad de Ingeniería, Ciencia y Tecnología. Universidad Bernardo O'Higgins, Santiago, Chile. claudio.gaete@ubo.cl 
to give bases for the design of school situations of socialization in higher education whose purpose is to incorporate these uses into the construction processes of the concept of defined integral, considering the functionality of mathematical knowledge, the epistemological plurality and the transversality of knowledge.

Key words: Modeling Category, Economy, Phenology, Socioepistemology, Uses of accumulation.

\section{INTRODUCCIÓN}

En relación a los procesos de enseñanza y aprendizaje del Cálculo, Aranda \& Callejo (2017a) indican que "muchas veces su presentación se focaliza en aspectos procedimentales como el manejo de reglas para calcular límites, derivadas o integrales" (p. 778). En el caso particular de la integral definida, "uno de los conceptos fundamentales del Cálculo" (Aranda \& Callejo, 2017b, p. 158), Martínez (2014) y Granera (2019) señalan que la enseñanza del concepto de integral definida suele centrarse en lo algorítmico.

Lo señalado anteriormente, son algunas evidencias que dan cuenta del carácter hegemónico del discurso Matemático Escolar (dME)', lo cual quiere decir que el dME impone ciertos significados, procedimientos y argumentaciones del conocimiento matemático asociado a este concepto, donde impera la justificación razonada, dejando de lado los usos de conocimiento matemático $(\mathcal{U}(C M))$ de la gente, lo que tiene como consecuencia una invisibilización de la pluralidad epistemológica (Gómez, 2015). Una muestra del carácter hegemónico del dME, en el caso del concepto de integral definida, se refleja en lo señalado por Cantoral (2003):

La integral de $f$ desde $a$ hasta $b$ puede entenderse de diferentes manerassegún el programa teórico que se considere. Consideremos, a manera de ejemplo, tres de las versiones más conocidas de la integral. La primera, la más usada en la enseñanza contemporánea para definir a la integral, se conoce como la integral de CauchyRiemann. Otra, la integral de Newton-Leibniz, es la más empleada al momento de resolver integrales por métodos elementales y finalmente, la menos conocida en la literatura escolar, la integral de Wallis. Esta integral fue tratada como parte de un programa tendiente a dar un tratamiento aritmético del infinito (p. 10).

Cantoral (2013) define el dME como un discurso que valida la introducción del saber matemático al sistema educativo. 
A pesar de existir, al menos, tres formas distintas de construir y entender el concepto de integral definida, Cantoral (2003) señala que se ha aceptado una especie de consenso escolar, donde la presentación de Cauchy-Riemann y la explicación mediante rectángulos inscritos y circunscritos, como medio de aproximación del área bajo la curva, es la que todos los profesores deben usar en sus clases.

Morales, Mena, Vera \& Rivera (2012) indican que existen estudios socioepistemológicos que muestran que lo importante para la construcción de la integral, dentro de situaciones de variación continua, es entender la noción de acumulación (Cordero, 2003a), en donde por medio de la práctica de predecir emerge una matemática funcional, vía un pensamiento variacional, entendiendo a la funcionalidad del conocimiento matemático como un conocimiento incorporado orgánicamente en el humano, que lo transforma y transforma su realidad; todo en oposición a la justificación razonada (Cordero, Gómez, Silva-Crocci \& Soto, 2015). Específicamente, esto es detallado en la llamada Socioepistemología del Cálculo y del Análisis (ver Tabla 1) en donde se da cuenta de que dentro de situaciones de variación, es posible construir el concepto de integral definida a partir de un procedimiento consistente en la comparación de dos estados, en donde el instrumento son las cantidades de variación continua y la argumentación es la predicción, lo cual permite significar dicho concepto a ideas relacionadas con flujo, movimiento, acumulación o estado permanente. A pesar de este hecho, una excesiva algoritmización en los procesos de enseñanza y aprendizaje de la integral definida no permite asociar y entender este concepto dentro de fenómenos de variación (Contreras \& Ordoñez, 2006, Cordero, 2005). Más aún, Morales et al. (2012) indican que situaciones de variación basadas en la situación núcleo de variación (ver Tabla 1) no están presentes en el dME. 
Tabla 1

Socioepistemología del Cálculo y el Análisis (Cordero, 2003b; Cordero, Del

Valle \& Morales, 2019, Cordero, Henríquez, Solis, Méndez, Opazo \& De la Cruz, 2019). Fuente: Cordero et al., 2019).

\begin{tabular}{|c|c|c|c|c|c|c|}
\hline \multirow[b]{2}{*}{$\begin{array}{l}\text { CONSTRUCCIÓN } \\
\text { DE LO } \\
\text { MATEMATICO }\end{array}$} & \multicolumn{6}{|c|}{ SITUACIONES } \\
\hline & VARIACIÓN & TRANSFORMACIÓN & APROXIMACIÓN & SELECCIÓN & PONDERACIÓN & PERIODIZACIÓN \\
\hline Significaciones & $\begin{array}{c}\text { Flujo } \\
\text { Movimiento } \\
\text { Acumulación } \\
\text { Estado } \\
\text { Permanente }\end{array}$ & $\begin{array}{c}\text { Patrones de } \\
\text { comportamiento } \\
\text { gráficos y } \\
\text { analiticos }\end{array}$ & $\begin{array}{c}\text { Límite } \\
\text { Derivación } \\
\text { Integración } \\
\text { Convergencia }\end{array}$ & $\begin{array}{l}\text { Patrón de } \\
\text { adaptación }\end{array}$ & $\begin{array}{l}\text { Distribución de } \\
\text { comportamiento }\end{array}$ & $\begin{array}{l}\text { Reproducción de } \\
\text { comportamientos }\end{array}$ \\
\hline Procedimientos & $\begin{array}{c}\text { Comparación } \\
\text { de dos } \\
\text { Estados }\end{array}$ & $\begin{array}{l}\text { Variación de } \\
\text { parámetros }\end{array}$ & $\begin{array}{l}\text { Operaciones } \\
\text { lógico formales } \\
\text { (cociente) }\end{array}$ & $\begin{array}{c}\text { Distinción de } \\
\text { cualidades }\end{array}$ & Equiparar & $\begin{array}{c}\text { Comparación de } \\
\text { periodos }\end{array}$ \\
\hline Instrumentos & $\begin{array}{l}\text { Cantidad de } \\
\text { variación } \\
\text { continua } \\
f(x+h)-f(x)=\cdot h \\
\cdot=f(x)\end{array}$ & $\begin{array}{c}\text { Instrucción que } \\
\text { organiza } \\
\text { comportamientos } \\
y=A f(B x+C)+D\end{array}$ & $\begin{array}{l}\text { Formas analiticas } \\
h \frac{f(x+h)-f(x)}{h}-f(x)\end{array}$ & Lo estable & $\begin{array}{l}\text { Punto de } \\
\text { equilibrio } \\
a_{1}-x=0\end{array}$ & Interpolación \\
\hline & Predicción & $\begin{array}{c}\text { Comportamiento } \\
\text { tendencial }\end{array}$ & $\begin{array}{l}\text { Analiticidad de } \\
\text { las funciones }\end{array}$ & Optimación & Compensación & 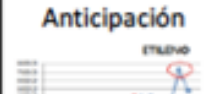 \\
\hline Resignificaciones & $E_{a}+\mathrm{V}$ & 0 & (x) & & $=$ & iIIII!iiitiiii \\
\hline
\end{tabular}

Nota. La socioepistemología del Cálculo y el Análisis da cuenta de seis situaciones núcleo específicas que formulan, individual y también conjuntamente, una epistemología del Cálculo, en donde la cuarta columna caracteriza la manera usual que tiene el dME de abordar las temáticas relacionadas al Cálculo, y en particular, del Cálculo Integral. Las significaciones corresponden a los elementos que le dan sentido a la situación específica, los procedimientos se entienden como una ejecución fundamental derivada de las significaciones y el instrumento se entiende como la experiencia sobre la cual se trabaja. Finalmente, se conciben las argumentaciones del conocimiento matemático "como el hilo conductor de la situación específica de donde emergen los conocimientos matemáticos" (Cordero et al., 2015, p. 74) 
De esta manera, se evidencia una problemática relacionada con los procesos de enseñanza y aprendizaje del concepto de integral definida, la cual apunta a la existencia, en el dME, del fenómeno de opacidad (Gómez, 2015) en el caso de los usos de la acumulación (U( $(a c)$ ), dentro de situaciones específicas de variación. Una manera de confrontar esta problemática, es formulando un marco de referencia ${ }^{2}$ que dé cuenta de dichos usos en situaciones específicas de variación, lo cual permitiría dar pautas para futuras intervenciones didácticas que contribuyan al abordaje de esta problemática. En ese sentido, la categoría de modelación ( $($ Mod)), variedad socioepistemológica de la noción de modelación matemática, resulta pertinente para el abordaje de esta problemática, ya que permite rescatar los $\mathcal{U}(a c)$, valorando la funcionalidad del conocimiento matemático y la pluralidad epistemológica, características que no suelen formar parte del dME.

De esta manera, nuestro objetivo de investigación se enfocará en desarrollar la $\zeta(\mathrm{Mod})$, es decir, analizar los $\mathcal{U}(a c)$ en situaciones específicas de variación, junto con las significaciones, procedimientos, instrumento y argumentación de cada una de estas situaciones. Para ello, se describe inicialmente el marco teórico en el cual se enmarca este trabajo, junto con la $\zeta(\mathrm{Mod})$ (sección 2). A continuación, se darán a conocer los objetivos de investigación en términos del marco teórico (sección 3) y los aspectos metodológicos que permitirán responder a dichos objetivos (sección 4), para posteriormente desarrollar la $\zeta(\mathrm{Mod})$ en dos situaciones de variación continua específicas (sección 5). Finalmente, se darán a conocer las conclusiones de esta investigación (sección 6).

\section{LA SOCIOEPISTEMOLOGÍA Y LA CATEGORÍA DE MODELACIÓN}

La socioepistemología es una teoría que busca enfocarse en los $\mathcal{U}(C M)$ de la gente, donde estos viven y se desarrollan: la escuela, el trabajo y la ciudad (Cordero et al., 2015). Esta teoría se ocupa del problema que plantea la conformación del saber matemático, asumiendo la legitimidad de toda forma de saber, sea este culto, técnico o popular (Cantoral, 2013), cada una con una verdad relativa (Cantoral, Reyes-Gasperini \& Montiel, 2014). Desde esta nueva

2 Entendemos marco de referencia como un constructo teórico socioepistemológico que "señala los elementos que comprenden las diferentes perspectivas y configuraciones posibles que indican pautas y trazan explicaciones sobre las maneras de construir y difundir, en este caso, la enseñanza y el aprendizaje del conocimiento matemático" (Gómez, 2015, p. 51). 
postura, "conocer será relativo a la circunstancia y al uso específico; es decir, depende del contexto y la situación" (Cordero et al., 2015, p. 89).

Esta teoría busca generar un marco de referencia que valore la justificación funcional, en donde al conocimiento matemático institucional se le incorporen los $\mathcal{U}(C M)$ de la matemática escolar, de otros dominios de conocimiento y del cotidiano de la gente. En cada uno de estos escenarios, se busca estudiar la resignificación de los usos de conocimiento matemático, entendido está como "la movilidad de los usos y significados del conocimiento matemático en las diferentes situaciones específicas propias de otros dominios de conocimiento y del cotidiano de la vida" (Mendoza \& Cordero, 2018, p. 37).

La socioepistemología propone el rediseño del dME, donde el núcleo principal sea una matemática funcional con base en los usos, lo que permite crear un vínculo entre la matemática del cotidiano y la de la escuela. Para tal finalidad, es importante dar cuenta de una epistemología de usos de conocimiento matemático, dado que "es la base para diseñar la situación escolar de socialización que trastoca y transforma la matemática escolar para crear la relación recíproca entre la matemática de la escuela y el cotidiano de las realidades" (Mendoza \& Cordero, 2018, p. 39). Cabe señalar que una epistemología de usos se conforma por las significaciones, procedimientos e instrumentos que generan la respectiva argumentación dentro de una determinada situación específica.

\subsection{La categoría de modelación}

Concordando con Williams \& Goos (2013), existe un enfoque tradicional de la modelación matemática en Educación, la cual consiste en reconocer a la práctica de modelación como un proceso cíclico (Blum \& Leiß, 2005, Blum \& Borromeo-Ferri, 2009), acuñando en muchas ocasiones a tránsitos y fases de la actividad individualizada (Blum et al., 2005, Maaß, 2006, Blomhøj, 2004, Lesh \& Doerr, 2003). Tales propuestas conciben una aproximación individualizada de la actividad de modelación (Borromeo-Ferri, 2006), incluso privilegiando contextos específicos como la modelación matemática en la Ingeniería (Rodríguez \& Quiroz, 2015). Ellos, son una influencia en currículos latinoamericanos (como han sido los casos de Chile, Perú, Colombia o Costa Rica, por mencionar algunos ejemplos) y demás latitudes. Tales trabajos respetan un principio, el que posiciona tanto a la realidad como a las matemáticas como unidades de conocimiento distanciadas, planteando una intención de ser relacionadas por medio de un proceso cíclico. Con la intención 
de sintetizar la manera en la que suele ser entendido, en términos generales, el concepto de modelación matemática, consideraremos un principio $P$ que acuñan tales aproximaciones teóricas, como el ciclo que conecta la realidad y las matemáticas.

Desde un enfoque socioepistemológico, la matemática no es concebida como un conocimiento ajeno a la realidad del que aprende. Por ello, al plantear la categoría de modelación, existe una necesidad por crear una variedad teórica en cuanto al principio que concilia el enfoque tradicional. Dicha variedad se ha ido construyendo y robusteciendo a partir de lo realizado en diferentes investigaciones socioepistemológicas (Cordero, 2017, Huincahue, 2017 y Cordero, Mena-Lorca, Huincahue, Mendoza \& Pérez-Oxté, 2019), en donde se considera una variación del principio $P$, digamos $P^{\prime}$, que considera la funcionalidad del conocimiento matemático en la práctica educativa. Específicamente, se concibe al principio $P^{\prime}$ como lo funcional de las relaciones reciprocas entre la matemática y el cotidiano (Cordero, 2017).

Plantear este principio como una variedad del enfoque tradicional, posiciona a la categoría de modelación $(\zeta(M o d)$ ) como un proceso descentrado del objeto matemático, más bien, cercano a un enfoque en el $\mathcal{U}(C M)$. Por ello, plantear la variedad $P^{\prime}$ permite romper con el modelo tradicional de islas entre la realidad y las matemáticas, posicionando a la $\zeta(M o d)$ como un modelo conceptual que privilegia la funcionalidad del conocimiento matemático, a partir del estudio de una $\operatorname{Res}(\mathcal{U}(C M))$.

Dado a lo anterior, consideraremos como objeto de estudio las actividades humanas que evidencian ciertos $\mathcal{U}(C M)$. Ellas, son inicialmente reconocidas a partir de una $\boldsymbol{i}$-ésima Situación Específica, $S_{i n}$, proveniente de un $n$-ésimo Dominio del Saber, $D_{n}$. Con respecto a las situaciones específicas, Cordero (2017) señala que "cada situación específica $S_{i}$ se conforma por elementos secuenciales que construyen lo matemático: significación, procedimiento, e instrumento, que derivan la argumentación de la situación $(\operatorname{Arg}(C M))$ (p. 18).

La relación entre $S_{i n}$ y $D_{n}$, está afectada por un eje epistemológico, digamos $E$, el cual, singulariza epistémicamente la actividad matemática realizada en $S_{i n}$, permitiendo destacar como foco de análisis la funcionalidad del conocimiento matemático. Este análisis, permite caracterizar los funcionamientos y formas del conocimiento matemático para una correcta descripción de los $\mathcal{U}(C M)$. En la Figura 1, se consideran, al menos, dos situaciones $S_{i n}$ y $S_{j m}$, reconociendo la pluralidad epistemológica del conocimiento matemático en distintos dominios (siendo $n$ y $m$ distintos o no) además, es importante indicar que $i$ 
es distinto de $\boldsymbol{j}$. Cabe destacar que la funcionalidad del saber matemático está afectada por la institucionalización que brinda el uso según la Comunidad de Conocimiento Matemático (Cordero, 2016, Pérez-Oxté, 2015) y la transversalidad del saber. Ambos componentes son ejes en donde suceden situaciones $S_{i n}$, caracterizadas respecto a $D_{n}$ y se crean oportunidades de alternancia de escenarios del tipo académico-escuela, la profesión-trabajo y el cotidiano-ciudad (Cordero, 2017).

El modelo de la Figura 1, representa los elementos que intervienen en la $\zeta(\operatorname{Mod})$, siendo ésta la $\operatorname{Res}(\mathcal{U}(C M))$ cuando suceden un tránsito entre $S_{\text {in }}$ y $S_{j m}$, incluso en alternancia de dominios. Este es el conocimiento que genera la $\zeta($ Mod) (Cordero, 2017).

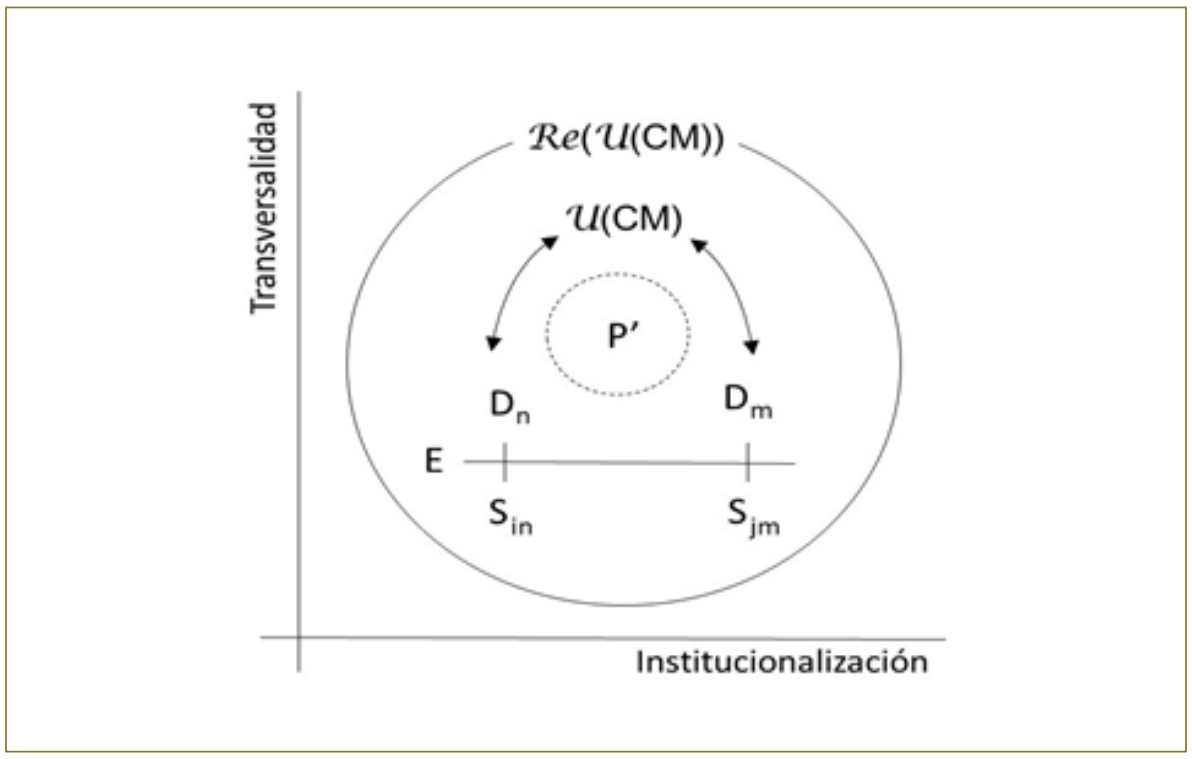

Figura 7. La ろ(Mod) (Cordero, Mena-Lorca, Huincahue, Mendoza \& Pérez-Oxté, 2019)

\section{OBJETIVOS DE INVESTIGACIÓN}

Para confrontar la problemática mencionada en la sección 1, a saber, que los $\mathcal{U}(a c)$ están opacados por el dME, es necesario dar cuenta de una $\operatorname{Res}(\mathcal{U}(a c))$ en situaciones específicas de variación. De esta forma, los objetivos de investigación, serán los siguientes: 


\subsection{Objetivo general}

Desarrollar la $\zeta(M o d)$ como una $\operatorname{Res}(\mathcal{U}(a c))$ en dos situaciones específicas de variación continua.

\subsection{Objetivo específico}

Desarrollar la $\zeta(\mathrm{Mod})$ como una $\operatorname{Res}(\mathcal{U}(\mathrm{ac}))$ en dos situaciones de variación continua específicas, denominadas cálculo teórico de la constante térmica y el cálculo teórico de los excedentes de los consumidores, propias de los dominios de conocimiento de la Fenología ${ }^{3}$ y la Economía, respectivamente.

\section{ASPECTOS METODOLÓGICOS}

Esta investigación estará guiada por el esquema metodológico para la investigación socioepistemológica (Montiel \& Buendía, 2012). Dicho esquema, está construido en base a las investigaciones realizadas por Buendía (2011) y Montiel (2011), el cual ha sido retroalimentado por diferentes investigaciones (e.g. Cordero, 2001, Castañeda, 2004, Molfino, 2010), junto con diversas discusiones dentro de la comunidad científica. En términos estructurales, este esquema (ver Figura 2) está compuesto de nodos, los cuales se conforman de un conjunto de tareas propias. Dichos nodos están, a su vez, unidos por medio de acciones relacionantes (representadas por flechas). Es importante señalar que una investigación que utilice este tipo de esquema metodológico no necesariamente debe abarcar la totalidad de nodos; dependiendo de los propósitos de investigación, puede estar compuesta de uno sólo o una combinación de estos, unidos por medio de ciertas acciones relacionantes.

3 Según la Real Academia de la Lengua Española, la Fenología es entendida como el "estudio de los fenómenos biológicos en relación con el clima, particularmente en los cambios estacionales". 


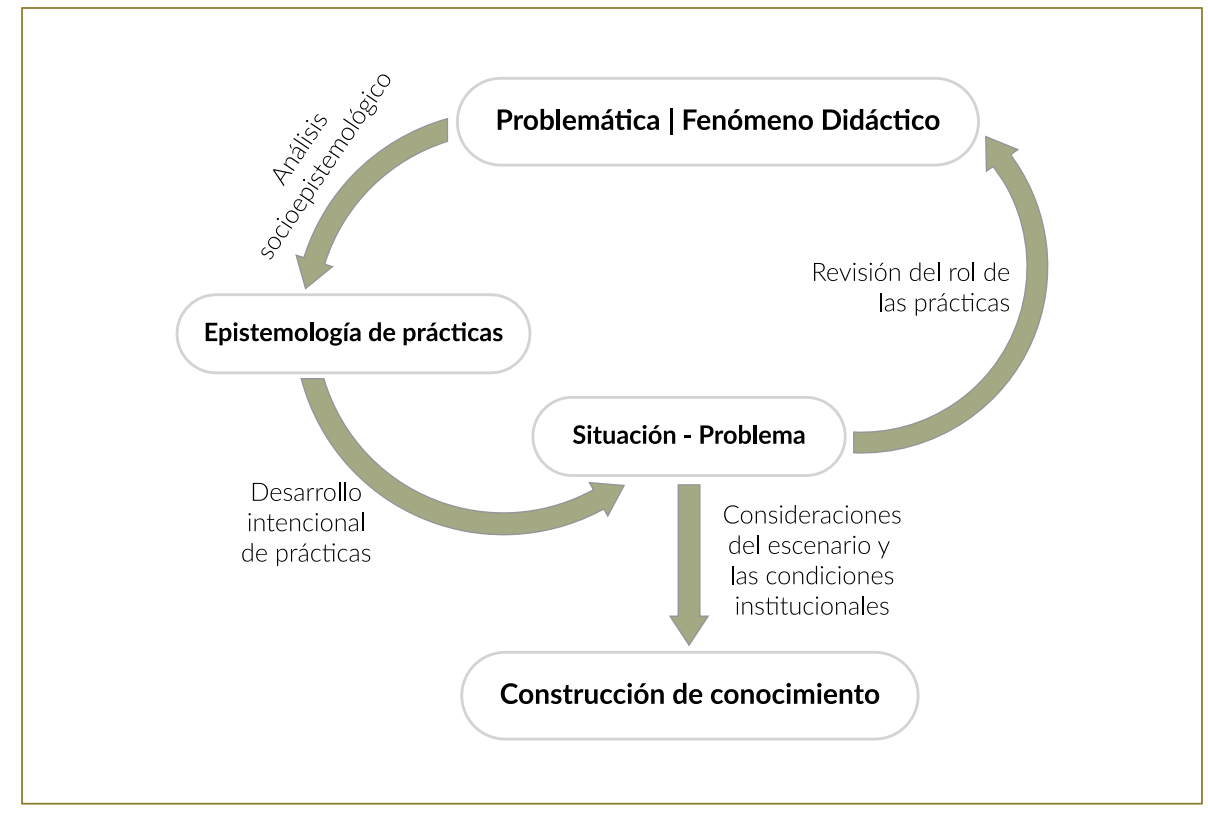

Figura 2. Esquema metodológico socioepistemológico (Montiel \& Buendía, 2012).

A partir del planteamiento de la problemática, así como los objetivos de investigación que permiten su abordaje, se realizará un análisis socioepistemológico, el cual estará conformado por dos dimensiones: la transmisión del saber y la resignificación del saber. En el caso de esta investigación, en la dimensión transmisión del saber se trató de estudiar brevemente la presentación del concepto de integral definida en el dME y permitió justificar la problemática de esta investigación (ver sección 1). Por medio de una técnica de análisis documental (Rojas, 2011), en la dimensión resignificación del saber se analizarán los $\mathcal{U}(a c)$ y las significaciones, procedimientos, instrumento y argumentación en las dos situaciones de variación continua denominadas cálculo teórico de la constante térmica y cálculo teórico de los excedentes de los consumidores, propias de los dominios de la Fenología y la Economía, respectivamente (ver sección 5).

\footnotetext{
4 Esta investigación entenderá al análisis documental como un "proceso de "inferencia" donde la información es estudiada, interpretada y sintetizada minuciosamente para dar lugar a una formulación que subyace al documento original" (Cordero et al., 2019, p. 193).
} 
Finalmente, dicho análisis nos permitirá proponer un desarrollo de la $\zeta(\operatorname{Mod})$ como una $\operatorname{Res}(\mathcal{U}(a c))$ en las situaciones específicas de variación anteriormente señaladas. De esta manera, esta investigación trabajará con los nodos Problemática/ Fenómeno didáctico y Epistemología de prácticas, unidos por la acción relacionante denominada Análisis socioepistemológico. La adaptación del esquema metodológico, para los propósitos de esta investigación, puede verse en la Figura 3.

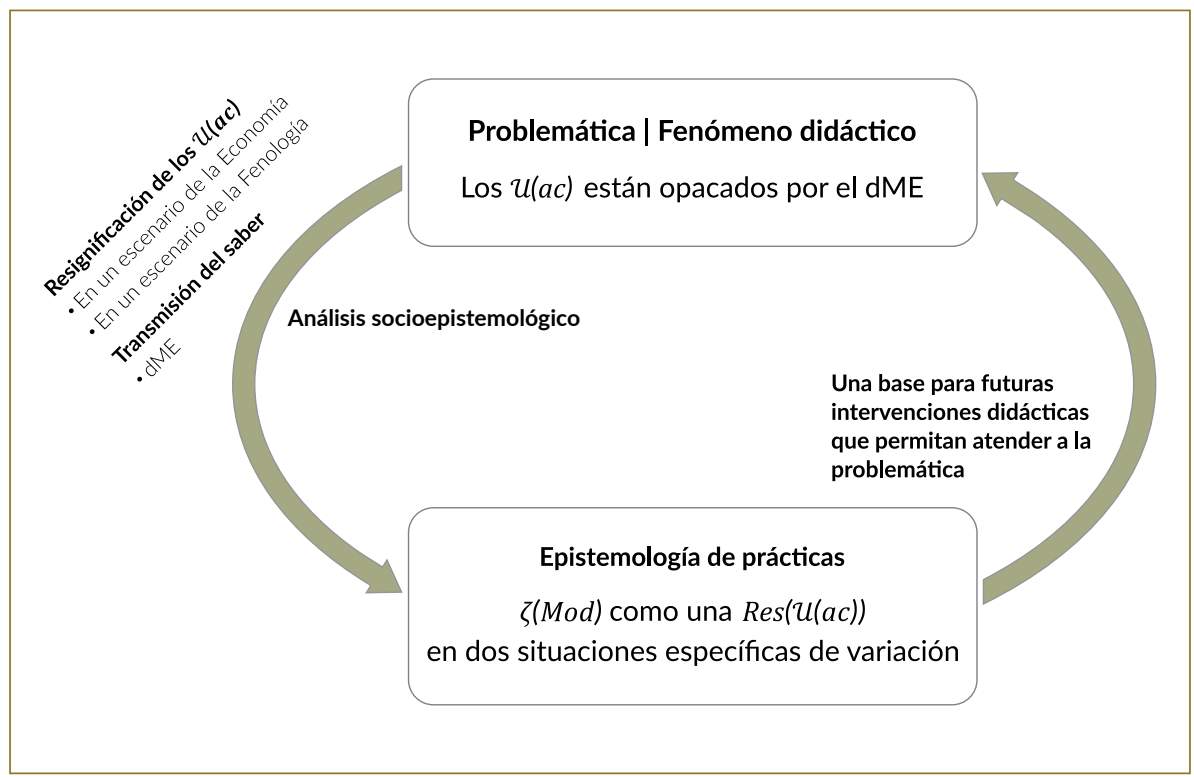

Figura 3. Adaptación del esquema metodológico para esta investigación.

Cabe mencionar que la manera de analizar los $\mathcal{U}(a c)$ en cada una de estas situaciones específicas, será acorde al análisis de $\mathcal{U}(\boldsymbol{C M})$ realizado en distintos trabajos socioepistemológicos (Del Valle, 2015, Morales \& Cordero, 2014, Mendoza, Cordero, Solís \& Gómez, 2018), los cuales analizan los $\mathcal{U}(C M)$ por medio de un debate entre funcionamiento y forma. 


\section{DESARROLLO DE LA $\zeta(M o d)$ COMO UNA $\operatorname{Res}(\mathcal{U}(a c))$}

\subsection{Resignificación de usos de la acumulación en Fenología}

Según Heuveldop, Pardo, Quirós \& Espinoza (1986), "la Fenología trata del estudio de los fenómenos o eventos biológicos periódicos en relación con los factores ambientales, principalmente las variaciones estacionales de las condiciones climáticas" (p. 171). Es sabido que la temperatura tiene incidencia en el desarrollo de muchos organismos. Parra-Coronado, Fischer \& ChavesCordoba (2015) indican que "la temperatura es una de las principales fuerzas impulsoras para el crecimiento y el desarrollo de los cultivos y varios estados fenológicos se manifiestan a través de su desarrollo" (p. 164). Para el caso específico de insectos y ácaros, el trabajo de Barrientos, Apablaza, Norero \& Estay (1998) destaca la sensibilidad de su desarrollo respecto a la temperatura en la que habita y su consecuente dependencia.

Al respecto, se hace necesario una unidad de medida que sea capaz de relacionar el desarrollo temporal de los ácaros y la temperatura en la cual ellos habitan, esto permitiría generar estudios con mayor precisión sobre los ciclos de vida de los animales. Tal unidad es Ilamada grados-días (Zalom, Godell, Wilson, Barnett \& Bentley, 1983), descrita como una unidad combinada de tiempo y temperatura, utilizada para medir el desarrollo o progreso de un organismo desde un punto a otro en su ciclo de vida (Huincahue, 2011). Al número de grados-días que han de ser acumulados para que ocurra un determinado evento fenológico, se le denomina constante térmica.

El concepto de grados-días ha sido ampliamente utilizado en la agricultura, especialmente para cuantificar y predecir eventos fenológicos. Según Rodríguez, Cotes \& Cure (2012), este concepto se ha utilizado en el análisis fenológico aplicado a diferentes tipos de cultivos, tanto en zonas templadas como en zonas tropicales, ya sea para insectos o ácaros. Urra \& Apablaza (2005) señalan que, en el caso del estudio de insectos, "el conocimiento de los grados-días provee una valiosa herramienta para el manejo de plagas, tanto para predecir infestaciones, programar medidas de manejo o realizar monitoreo" (p. 19). Sin embargo, no es una regla que se cumple para todos los ciclos de vida. Un caso de interés es el del Brevipalpus chilensis (B. chilensis), ya que es un ácaro sensible a débiles variaciones de temperaturas (Huincahue, 2011), por lo tanto, es de relevancia la precisión del cálculo de grados-días.

En Castillo y Santibáñez (1987), se encuentra una manera teórica de calcular la constante térmica: 


$$
L=\int_{t_{0}}^{t_{f}}\left(T-T_{h}\right) d t
$$

en donde, $T$ es la temperatura media; $T_{h}$ es la temperatura umbral inferior; $t_{0}$ es la fecha de inicio de la etapa de desarrollo y $t_{f}$ es la fecha de término de la etapa de desarrollo. En este caso, podemos establecer que $\left(T-T_{h}\right) d t$ representa la cantidad de grados-días acumulados en un determinado intervalo de tiempo $[t, t+d t]$, con $T-T_{h}=0$ si $T<T_{h}$. La temperatura umbral inferior es la temperatura en la cual el desarrollo del organismo en cuestión se detiene por el frío. A medida que la temperatura aumenta por encima de la temperatura umbral, el desarrollo se acelera hasta alcanzar una temperatura óptima, la cual se entiende como aquella en la cual el desarrollo ocurre lo más rápidamente posible. Este fenómeno es descrito en la Figura 4.

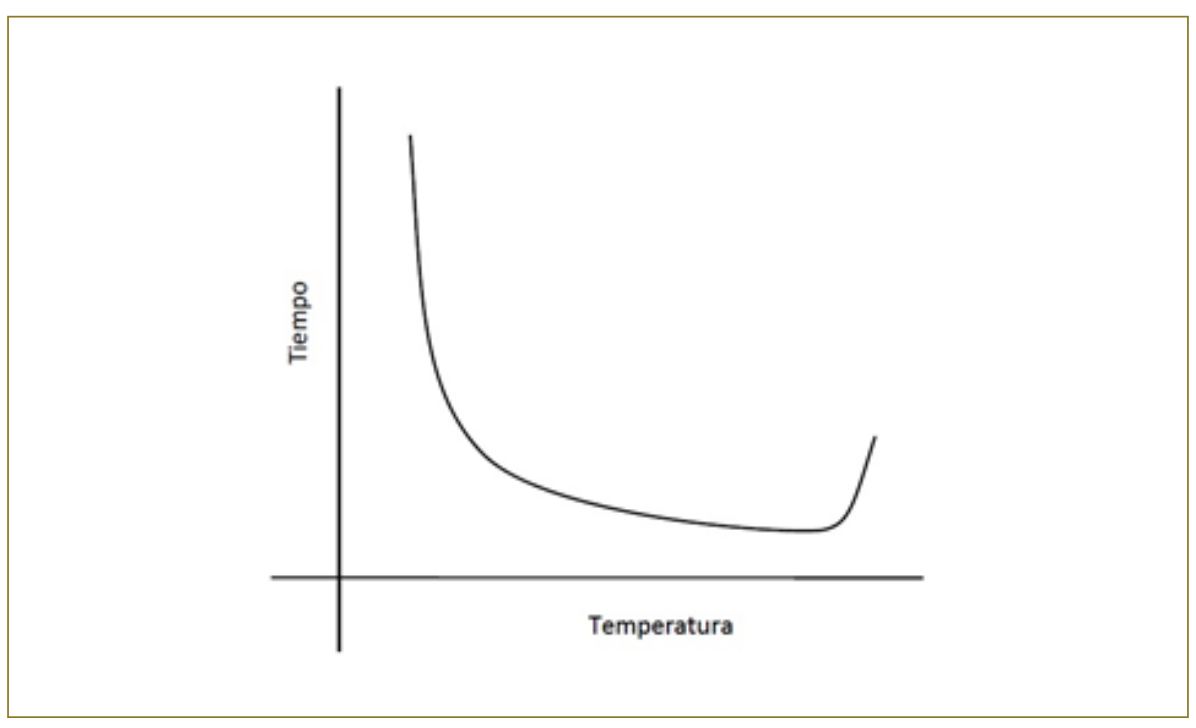

Figura 4. Curva de temperatura versus tiempo de un organismo que depende de la temperatura (Zalom, Godell \& Willson, 1983).

A partir de lo analizado en esta situación específica de variación, que denominamos cálculo teórico de la constante térmica, hemos logrado dar cuenta de una $\operatorname{Res}(\mathcal{U}(a c))$ al inferir tanto el $\mathcal{U}(a c)$ como su respectiva epistemología de $\boldsymbol{u}(a c)$.

En cuanto al $\mathcal{U}(a c)$, su funcionamiento es para determinar la cantidad de grados-días acumulados en un cierto intervalo de tiempo y su forma 
es por medio de la resta $A(t+d t)-A(t)$, donde $A(t)$ corresponde a los gradosdías acumulados en el tiempo $t$. Con respecto a la epistemología de $\mathcal{U}(a c)$, esta se conforma por las significaciones, procedimientos, instrumentos y argumentación, basadas en la segunda columna de la Tabla 1: la significación asociada es la de constante térmica, el procedimiento corresponde a la comparación de dos estados: $A(t+d t)-A(t)=\left(T-T_{h}\right) d t$, donde $T-T_{h}=0$ si $T$ $<T_{h}$, comparación que es posible de realizar sólo si $T$ varía de forma continua con respecto al tiempo (instrumento útil al humano), lo cual ocurre en esta situación, permitiendo predecir, como argumentación de esta situación, cuándo ocurrirá un determinado evento fenológico.

\subsection{Resignificación de usos de la acumulación en Economía}

El excedente individual de un consumidor se entiende como la resta entre lo que dicho consumidor está dispuesto a pagar por un producto, menos lo que terminó pagando (Krugman \& Wells, 2007). A partir de lo anterior, el Excedente de los Consumidores (EC) se define como la suma de todos los excedentes individuales. A partir de lo anterior, dichos autores señalan que los Excedente de los Consumidores (EC) corresponden a la suma de todos los excedentes individuales.

Los EC representan la ganancia monetaria de aquellos consumidores que son capaces de comprar un producto a un precio mayor al de mercado. Según Escribano (2001), el EC mide "el bienestar que se queda en manos del consumidor (excedente) por pagar un precio uniforme por todas las unidades consumidas, cuando en realidad el consumidor estaría dispuesto a pagar precios más altos por las primeras unidades consumidas" (p. 16). Para Mankiw (2004), el EC mide el beneficio de los compradores de un determinado producto tal como ellos lo perciben, siendo estos excedentes "una buena medida del bienestar económico si los responsables de la política económica quieren respetar las preferencias de los compradores" (Mankiw, 2004, p. 90). La medición de los EC es un elemento clave para el análisis costo-beneficio, transformándose en una técnica formal que permite ponderar los beneficios de un determinado proyecto público frente a sus costos. Según Frank (2009), los EC pueden ser utilizados para medir el impacto de políticas públicas.

Por la ley de oferta y demanda, las curvas de oferta y demanda deben ser crecientes y decrecientes, respectivamente (Mankiw, 2004). En la Figura 5, podemos notar que la franja vertical tiene área $p \Delta q$, siendo interpretado como la cantidad total de dinero que los consumidores 
gastarán comprando $\Delta q$ unidades del producto cuando el precio unitario es $p=p(q)$. Si el punto de equilibrio (punto en donde la oferta coincide con la demanda) es $\left(p_{0}, q_{0}\right)$, se tiene que dichos consumidores solo gastan $p_{0} \Delta q$ por estas $\Delta q$ unidades. Por lo tanto, se benefician en la cantidad $B_{C}(q+\Delta q)-B_{C}(q)=B_{C}(\Delta q) \approx p \Delta q-p_{0} \Delta q=\left(p-p_{0}\right) \Delta q$, donde $B_{C}(q)$ representa la suma de los excedentes individuales de todos los consumidores dispuestos a pagar un precio mayor a $p_{0}$, cuando la demanda es de $q$ unidades. Sumando las áreas de todos estos rectángulos desde $q=0$ hasta $q=q_{0}$, se tiene que el EC será:

$$
E C=\int_{0}^{q_{0}}\left(p-p_{0}\right) d q
$$

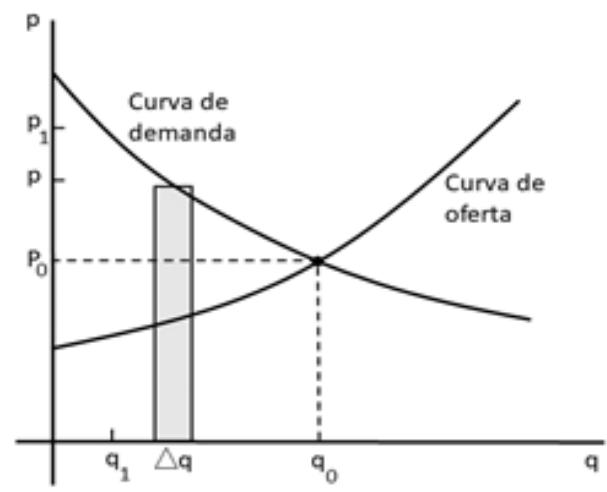

Figura 5. Curvas de oferta y demanda de un producto.

A partir de lo analizado en esta situación específica de variación, que denominamos cálculo teórico de los excedentes de los consumidores, se ha logrado dar cuenta de una $\operatorname{Res}(\mathcal{U}(a c))$ al inferir tanto el $\mathcal{U}(a c)$ como su respectiva epistemología de $\mathcal{U}(a c)$. En cuanto al $\mathcal{U}(a c)$, su funcionamiento es para estimar el beneficio, por la compra de las $\Delta q$ unidades comprendidas en el intervalo $[q, q+\Delta q]$, de aquellos consumidores dispuestos a pagar un precio mayor al de mercado y la forma de la acumulación, es por medio de la diferencia entre $B_{C}(q+\Delta q)$ y $B_{C}(q)$. Con respecto a la epistemología de $\mathcal{u}(a c)$, esta se conforma por las significaciones, procedimientos, instrumentos 
yargumentación, basadas en la segunda columna de la Tabla 1: la significación asociada es la de EC, el procedimiento corresponde a la comparación de dos estados: $B_{C}(x+d x)-B_{C}(x)=\left(p(x)-p_{0}\right) d x$, comparación que es posible de realizar sólo si $B_{C}(x)$ varía de forma continua (instrumento útil al humano), lo cual ocurre en esta situación, permitiendo predecir cuál será el excedente de aquellos consumidores que están dispuestos a pagar, por la compra de cierto producto, un precio mayor al que se fije en el mercado.

En la Tabla 2 se resumen las epistemologías de $\mathcal{U}(a c)$ en las dos situaciones específicas descritas anteriormente, las cuales se rigen por la situación núcleo de variación.

Tabla 2

Epistemologías de $\mathcal{U}(a c)$ construidas con base en la situación núcleo de variación.

Situación específica Situación especifica

\begin{tabular}{|c|c|c|}
\hline & Situación específica & Situación especifica \\
\hline $\begin{array}{l}\text { Construcción de lo } \\
\text { matemático }\end{array}$ & $\begin{array}{c}\text { Cálculo teórico de la constante } \\
\text { térmica }\end{array}$ & $\begin{array}{l}\text { Cálculo teórico de los Excedentes de los } \\
\text { Consumidores }\end{array}$ \\
\hline Significaciones & Constante térmica & Excedentes de los consumidores \\
\hline Procedimientos & $\begin{array}{c}\text { Comparación de dos } \\
\text { estados } \\
\begin{array}{c}A(t+d t)-A(t)=\left(T-T_{h}\right) d t \\
\text { donde } T-T_{h}=0 \text { si } T<T_{h}\end{array}\end{array}$ & $\begin{array}{l}\text { Comparación de dos estados } \\
B_{C}(x+d x)-B_{C}(x)=\left(p(x)-p_{0}\right) d x\end{array}$ \\
\hline $\begin{array}{l}\text { Instrumento útil al } \\
\text { humano }\end{array}$ & Cantidad de variación continua & Cantidad de variación continua \\
\hline Argumentación & $\begin{array}{l}\text { Predecir cuándo ocurrirá un } \\
\text { determinado evento fenológico }\end{array}$ & $\begin{array}{c}\text { Predecir el beneficio total de aquellos } \\
\text { consumidores dispuestos a pagar un } \\
\text { precio mayor al de mercado. }\end{array}$ \\
\hline
\end{tabular}




\section{CONCLUSIONES}

La problemática evidenciada en esta investigación fue que el dME opaca los $\mathcal{U}(a c)$. Para confrontar dicha problemática, se planteó como objetivo general el desarrollar la $\zeta(M o d)$ como una $\operatorname{Res}(\mathcal{U}(a c))$ en dos situaciones específicas de variación. La primera $\operatorname{Res}(\mathcal{U}(a c))$ ocurrió en el escenario de la Fenología, en donde la situación específica se denominó cálculo teórico de la constante térmica y la segunda $\operatorname{Res}(\mathcal{U}(a c))$ ocurrió en el escenario de la Economía, en donde la situación específica se denominó cálculo teórico de los excedentes de los consumidores.

En el escenario de la Fenología, se ofreció una $\operatorname{Res}(\mathcal{U}(a c))$ al problematizar la predicción en la que ocurrirá determinado evento fenológico. En este caso, el $\mathcal{U}(a c)$ emergió al momento de determinar la acumulación de grados-días durante un intervalo de tiempo $[t, t+d t]$. Además, dicha categoría permitió darle un significado a la integral definida: como el de constante térmica.

En el escenario de la Economía, se ofreció una $\operatorname{Res}(\mathcal{U}(a c))$ al problematizar la predicción del beneficio total que tendrían aquellos consumidores que están dispuestos a pagar, por la compra de cierto artículo, un precio mayor al precio de mercado. En este caso, el $\mathcal{U}(a c)$ emergió al momento de determinar cuánto fue el beneficio de este tipo de consumidores por la compra de las unidades comprendidas en el intervalo $[x, x+d x]$. Además, esta categoría permitió darle un significado a la integral definida: como excedentes de los consumidores, cuya interpretación geométrica corresponde al área comprendida entre la curva de demanda $p=p(x)$ y la recta $p=p_{0}$, donde $p_{0}$ corresponde al precio unitario de mercado de cierto producto.

Las epistemologías de $\mathcal{U}(a c)$ entregadas en esta investigación, además de ser de una naturaleza diferente a lo establecido en el dME, aportan a la socioepistemología nuevas epistemologías basadas en la situación núcleo de variación (ver Tabla 2), las cuales, a su vez, otorgan bases para el diseño de situaciones escolares de socialización, en el sentido de Gómez (2015), contribuyendo a trastocar y transformar la matemática escolar con el propósito de generar una relación recíproca entre el cotidiano de la gente y la matemática escolar (Mendoza \& Cordero, 2018), tomando en cuenta la funcionalidad del conocimiento matemático. Por lo tanto, la inclusión de la noción de acumulación en los procesos de enseñanza y aprendizaje del cálculo integral es una condición necesaria para contribuir al logro de la reciprocidad entre la matemática escolar y la matemática del cotidiano. Sin embargo, esto por sí solo no resulta suficiente; también es necesario que esta 
noción se acepte como un producto material social que debe de enseñarse y aprenderse.

Desarrollar la $\zeta($ Mod $)$ como una $\operatorname{Res}(\mathcal{U}(a c))$ contribuye a favorecer el aprendizaje de significados del conocimiento matemático asociado a la integral definida, por medio de un enfoque en los $\mathcal{U}(a c)$, privilegiando la funcionalidad del conocimiento matemático, la pluralidad epistemológica y la transversalidad de saberes.

Finalmente, para robustecer la $\zeta(\mathrm{Mod})$ presentada en esta investigación, resulta fundamental diseñar dos situaciones de aula, basadas en las epistemología de $\mathcal{U}(a c)$ presentadas en la Tabla 2, y analizar, a partir de la implementación de dichos diseños, cómo estudiantes de educación superior logran, tanto construir el concepto de integral definida en cada una de las situaciones, como otorgar a dicho concepto significados diferentes.

\section{REFERENCIAS BIBLIOGRÁFICAS}

Aranda, C., \& Callejo, M. (2017a). Construcción de la Función Integral y Razonamiento Covariacional: dos Estudios de Casos. Bolema, 37(18), 777-798.

Aranda, C., \& Callejo, M. (2017b) Formas de aproximar el área bajo una curva: un estudio con estudiantes de bachillerato. Enseñanza de las Ciencias, 35(1), 157-174.

Barrientos, R., Apablaza, J., Norero, H., \& Estay, P. (1998). Temperatura base y constante térmica de desarrollo de la polilla del tomate, tuta absoluta (lepidoptera: gelechiidae). Ciencia e Investigación Agraria, 25(3), 133 $-137$.

Blomhøj, M. (2004). Mathematical Modelling - A Theory for Practice. En B. Clarke et al. (Eds.), International Perspectives on Learning and Teaching Mathematics (pp. 145-159). Göteborg: National Center for Mathematics Education.

Blum, W., \& Leiß, D. (2005). "Filling up" - The Problem of Independence-Preserving Teacher Interventions in Lessons with Demanding Modelling Tasks. En Bosch, M. (Ed.), CERME-4 - Proceedings of the Fourth Conference of the European Society for Research in Mathematics Education (pp. 1623-1633). IQS FUNDIEMI Business Institute. 
Blum, W., \& Borromeo-Ferri, R. (2009). Mathematical Modelling: Can It Be Taught And Learnt? Journal of Mathematical Modelling and Application, 7(1), 45-58.

Borromeo-Ferri, R. (2006). Theoretical and empirical differentiations of phases in the modelling process. ZDM - The International Journal on Mathematics Education, 38(2), 86-95.

Buendía, G. (2011). La construcción social del conocimiento matemático escolar. Un estudio socioepistemológico sobre la periodicidad de las funciones. México: Ediciones Diaz de Santos.

Cantoral, R. (2003). La aproximación socioepistemológica a la investigación en matemática educativa: una mirada emergente [CD-ROM]. XI Conferencia Interamericana de Educação Matemática (tema: Educación Matemática y Desafíos y Perspectivas). Brazil, Blumenau: Universidad Regional de Blumenau.

Cantoral, R. (2013). Teoría Socioepistemológica de la Matemática Educativa. Barcelona: Gedisa.

Cantoral, R., Reyes-Gasperini, D., \& Montiel, G. (2014). Socioepistemología, Matemáticas y Realidad. Revista Latinoamericana de Etnomatemática, 7(3), 91-116. Recuperado de http://www.redalyc.org/ pdf/2740/274032530006.pdf

Castañeda, A. (2004). Un acercamiento a la construcción social del conocimiento: estudio de la evolución didáctica del punto de inflexión (Tesis doctoral). CICATA-IPN, México.

Castillo, H., \& Santibáñez, F. (1987). Efecto de la temperatura sobre la fenología del trigo. Agricultura Técnica (Chile), 47 (1), 29 -34.

Contreras, Á., \& Ordóñez, L. (2006). Complejidad ontosemiótica de un texto sobre la introducción a la integral definida. Relime, 9(1), 65-84. Recuperado de http://www.scielo.org.mx/pdf/relime/v9n1/v9n1a4.pdf

Cordero, F. (2001). La distinción entre construcciones del cálculo. Una epistemología a través de la actividad humana. Revista Latinoamericana de Matemática Educativa, 4(2), 103-128. 
Cordero, F. (2003a). Reconstrucción de significados del Cálculo Integral. La noción de acumulación como una argumentación. México: Grupo Editorial Iberoamérica.

Cordero, F. (2003b). Lo social en el conocimiento matemático: los argumentos y la construcción de significados. Acta Latinoamericana de Matemática Educativa, 16, 73-78.

Cordero, F. (2005). El rol de algunas categorías del conocimiento matemático en educación superior. Una socioepistemología de la integral. Relime, 8(3), 265-286.

Cordero, F. (2016). Modelación, funcionalidad y multidisciplinaridad: el eslabón de la matemática y el cotidiano. En J. Arrieta y L. Díaz (Eds.), Investigaciones latinoamericanas de modelación de la matemática educativa (pp. 59-88). Barcelona, España: Gedisa.

Cordero, F (2017). La matemática y lo matemático. Transversalidad y modelación: un programa socioepistemológico. Artículo en preparación.

Cordero, F., Del Valle, T., \& Morales, A. (2019). Usos de la optimización de ingenieros en formación: el rol de la Ingeniería mecatrónica y de la obra de Lagrange. Revista Latinoamericana de Investigación en Matemática Educativa, 22(2), 185-212. Doi: https://doi.org/10.12802/relime.19.2223

Cordero, F., Gómez, K., Silva - Crocci, H., \& Soto, D. (2015). El discurso matemático escolar: la adherencia, la exclusión y la opacidad. México: Gedisa.

Cordero, F., Henríquez C., Solis, M., Méndez, C., Opazo, C., \& De la Cruz, A. (2019). La modelación en la Matemática Educativa: sus programas de investigación y la docencia. El rol de la transversalidad de saberes matemáticos. Revista Acta Latinoamericana de Matemática Educativa, 32(7), 540-547.

Codero, F., Mena-Lorca, J., Huincahue, J., Mendoza, J., \& Pérez-Oxté, I. (2019). A category of modeling: the uses of mathematical knowledge in different scenarios and the learning of mathematics. Artículo en preparación.

Del Valle, T. (2015). Los Usos de la Optimización: Un Marco de Referencia y la Teoría Socioepistemológica (Tesis doctoral). Pontificia Universidad Católica de Valparaíso, Chile. 
Escribano, A. (2001). El funcionamiento de los mercados y el comercio electrónico. Principios básicos para el análisis. Economía Industrial, 340(4), 13-30.

Frank, R. (2009). Microeconomía intermedia análisis y comportamiento económico. México: Mc Graw Hill.

Gómez, K. (2015). El fenómeno de opacidad y la socialización del conocimiento. Tesis de Doctorado no publicada, Centro de investigación y de Estudios Avanzados del Instituto Politécnico Nacional, D.F., México.

Granera, J. (2019). La integral definida como el área bajo la curva en un entorno computacional. Revista Científica de FAREM-Estelí, 8(30), 3-19. Doi: https://doi.org/10.5377/farem.v0i30.7883

Heuveldop, J., Pardo, J., Quirós, S., \& Espinoza, L. (1986). Agroclimatología Tropical. San José, Costa Rica: Editorial Universidad Estatal a Distancia.

Huincahue, J. (2011). Dinámicas de modelos de Depredación Continuos e Impulsivos y Estudio Fenológico del Brevipalpus Chilensis (Tesis de Maestría no publicada). Instituto de Matemáticas, Pontificia Universidad Católica de Valparaíso, Chile.

Huincahue, J. (2017). Propuesta de modelación matemática en la formación de profesores y bases para una variedad de modelación desde la teoría Socioepistemológica. (Tesis doctoral). Pontificia Universidad Católica de Valparaíso, Chile.

Krugman, P., \& Wells, R. (2007). Macroeconomía: introducción a la Economía. Barcelona: Reverté.

Lesh, R., \& Doerr, H. (Eds.) (2003). Beyond Constructivismen-Models and Modeling Perspectives on Mathematics Problem Solving, Learning and Teaching. Mahwah: Lawrence Erlbaum.

Maaß K. (2006). What are modelling competences? ZDM-The International Journal on Mathematics Education, 38(2), 113-142.

Mankiw, N. (2004). Principios de Economía. (3a Ed.). México: McGraw Hill

Martínez, F. (2014). Recursos para el cálculo visual de integrales. Educación Matemática, 26(1), 153-169. Recuperado de http://somidem.com.mx/ descargas/Vol26-1-6.pdf 
Morales, A., Mena, J., Vera, F., \& Rivera, R. (2012). El rol del tiempo en un proceso de modelación utilizando videos de experimentos físicos. Enseñanza de las Ciencias, 30(3), 237-256.

Morales, A., \& Cordero, F. (2014). La graficación-modelación y la Serie de Taylor. Una socioepistemología del Cálculo. Revista Latinoamericana de Investigación en Matemática Educativa, 17(3), 319-345. DOI: https:// dx.doi.org/10.12802/relime.13.1733

Mendoza, J., \& Cordero, F. (2018). La modelación en las comunidades de conocimiento matemático. El uso de las matemáticas en ingenieros biónicos. El caso de la estabilidad. Revista Latinoamericana de Etnomatemática, 77(1), 36-61.

Mendoza, J., Cordero, F., Solís, M., \& Gómez, K. (2018). El Uso del Conocimiento Matemático en las Comunidades de Ingenieros. Del Objeto a la Funcionalidad Matemática. Bolema, 32(62), 1219-1243.

Molfino, V. (2010). Procesos de institucionalización del concepto de límite: un análisis socioepistemológico. (Tesis doctoral). CICATA-IPN, México.

Montiel, G. (2011). Construcción de conocimiento trigonométrico. Un estudio socioepistemológico. México: Ediciones Díaz de Santos.

Montiel, G., \& Buendía, G. (2012). Un esquema metodológico para la investigación socioepistemológica: Ejemplos e ilustraciones. En A. Rosas y A. Romo (Eds.), Metodología en Matemática Educativa: Visiones y reflexiones (pp. 55-82). México: Lectorum.

Parra-Coronado, A., Fischer, G., \& Chaves-Cordoba, B. (2015). Tiempo térmico para estados fenológicos reproductivos de la feijoa (Acca sellowiana (O. Berg) Burret). Acta biológica. Colombiana, 20(1), 163-173.

Pérez-Oxté, I. (2015). Los usos de la gráfica en una Comunidad de Ingenieros Químicos Industriales en Formación. Una base para el diseño de una situación de aprendizaje (Tesis de Maestría no publicada). Centro de Investigación y de Estudios Avanzados del Instituto Politécnico Nacional. México.

Rodríguez, D., Cotes, J., \& Cure, J. (2012). Comparison of eight degree-days estimation methods in four agroecological regions in Colombia. Bragantía, Campinas, 71(2), 299-307. 
Rodríguez, R., \& Quiroz, S. (2015). Developing modelling competencies through the use of technology. En Stillman, Blum \& Biembengut (Eds.). Mathematical Modelling in Education Research and Practice. Australia: Springer International Publishing.

Rojas, I. (2011). Elementos para el diseño de técnicas de investigación: Una propuesta de definiciones y procedimientos en la investigación científica. Tiempo de Educar, 12(24), 277-297.

Urra, F., \& Apablaza, J. (2005). Temperatura Base y Constante Térmica de Desarrollo de Copitarsia decolora (Lepidoptera: Noctuidae). Ciencia e Investigación Agraria, 32(1), 19-26.

Williams, J., \& Goos, M. (2013). Modelling with mathematics and technologies. En M. A. Clements, A. J. Bishop, C. Keitel, J. Kilpatrick y F. K. S. Leung (Eds.), Third international handbook of mathematics education (pp. 549-569). Berlin, Alemania: Springer.

Zalom, F., Godell, P., Wilson, L., \& Barnett, W. (1983). Degree-days: The calculation and use of heat units in pest management. Division of Agriculture and Natural Resources, 2-10. 